\title{
Study on Autonomous Path Planning by Mobile Robot for Road Nondestructive Testing Based on GPS
}

\author{
Lunhui $\mathrm{Xu}^{1}$, Fan $\mathrm{Ye}^{2}$, and Yanguo Huang ${ }^{1}$ \\ ${ }^{1}$ Faculty of Mechanical and Electronic Engineering, \\ Jiangxi University of Science and Technology, Ganzhou, P. R. China, 341000 \\ ${ }^{2}$ South China University of Technology of Traffic Collage, Guangzhou, P. R. China, 510006 \\ 1hxu@scut.edu.cn, yefan24@163.com, huangyanguo2004@126.com
}

\begin{abstract}
This paper analyses the statements of video imaging, CT, infrared thermography and radiography applied in the road nondestructive testing, and design a path planning mobile robot with GPS positioning which can remarkably increase the efficient of road nondestructive testing. Besides, appropriate algorithm for nondestructive testing on the road autonomous mobile robot path planning is given. This method is simplicity, versatility, and efficiency. The mobile robot are selected for example and the simulation results represent the effectiveness of this method.
\end{abstract}

Keywords: road nondestructive testing, GPS, path planning, mobile robot.

\section{Introduction}

Because of the natural factor and long-term used, roads may be damaged. The damaged road is harmful to the safety of vehicles, so the regular road testing is necessary.

While testing the road's structure, the destructive testing may descend the traffic capacity and waste a lot of money and time. So, to meet the requirements of road development and ensure road traffic safety, nondestructive testing (NDT) is becoming more and more important. But the traditional method of road nondestructive testing is manual operation, which can only carry out a limited number of testing, and may not achieve long-term monitoring. In addition, traffic flow on major highways, testing personnel may be braved danger. Therefore, making the manual testing developed to automated testing technology, and low-speed, low accuracy developed to high-speed, high precision is critical important. So, a fast, accurate and nondestructive road testing method is needed urgently.

The constant improvement of science and technology promotes the growth of mobile robot for road nondestructive testing. Instead of human beings, mobile robot for road nondestructive testing can suffer a large number of works with strong intensity, long time, high repeatability and terrible environment. These objective requirements propel the widely development in the study of mobile robot.

Path planning is one of the important core problem and technique in the research area of mobile robot that not only develops fast but also contributes a lot [1]. Path planning by mobile robot refers that it can search for the best (or second-best) and reasonable moving strategy from the initial status (including position and angle) to the 
final status by following the planed path. It is the foundation for the mobile robot to execute different kinds of tasks. In this paper, appropriate algorithm for nondestructive testing on the road autonomous mobile robot path planning is given . The feasibility of the scheme and algorithm proposed in this paper was verified by the experimental prototype test data.

\section{Current Statements of Road NDT}

Currently, the major testing methods of nondestructive testing methods are including video imaging, CT, infrared thermography and radiography. The introductions of all of the methods are as follows:

\subsection{Video Imaging}

Video imaging systems have become versatile data-gathering tools because of their high spatial resolution, near real-time image availability and computer compatibility. Current video imaging is widely used for non- Destructive testing. There are a lot of digital video imaging systems which can be used for the pavement broken testing such as PAVUE and WiseCrax [2].

These systems are widely used for Nondestructive testing; the steps of using these systems are as flows:

1. Get the image of the pavement, and then input this digital images into computer;

2. Change the consecutive images into still images, use the image processing technology to analyze and classify the still images.

3. Evaluate the road damage level and parameter such as crack breadth, length and square and so on. Meanwhile, according to the GPS, passion the damage section.

\subsection{Computed Tomography (CT)}

In highway construction, to ensure the quality of engineering, relative mass detection is needed in a wide range including tamping foundation, rolling sub grade and paving pavement. The traditional detection is to on-site observe the roads and sample from drilling for the laboratory analysis, so that the control parameter of engineering quality, such as thickness, depth, degree of compaction and strength, can be obtained. However, this routine method has some limitation. First, it can be omitted easily although the measured points are selected randomly so that they should be representative to some extent. Second, the problems about the damage of the pavement, such as crack and sag, can be easily found, while the inner problems of the roads are difficult to find out. Therefore, some nondestructive, fast and direct test equipment and technique that can show the inner status of the roads is necessary for the improvement of highway construction and maintenance level.

CT was invented in 1972. In the early stage it was only applied in X-ray, then the application was widely extended, including emission computed tomography (ECT), ultrasound computed tomography, magnetic resonance imaging (MRI) and so on. 
This technique was at first applied in the field of biomedicine. The basic method of CT is image reconstruction, which refers to the reconstruction of the cross-section image obtained from projections of measured objects by the computer. Application of $\mathrm{CT}$ in road quality testing is like perspective diagnosis of the roads, which can help us to detect the diseases promptly and have a direct view. This technique has been used in the pavement detection by ground penetrating radar. The road radar, which is based on the use of transient electromagnetic waves, emits high-frequency spike pulse electromagnetic waves to the underground by wide time-domain transmitting antenna. When waves meet different interfaces of dielectric during the underground transmission, reflection happens. Then it is received by receiving antenna and handled by the computer, which can analyze the obtained digital signal, so that we can get three dimensional computed tomography imaging for the underground target. Accordingly, we are able to clearly observe the cavity of sub grade, thickness and deformation of the pavement structural layer, even the water under the surface.

\subsection{Infrared Thermography (IRT)}

Infrared thermography (IRT) has established as a fast and reliable tool in many areas of non destructive testing. In recent years, IRT has emerged as a widely used method for road nondestructive testing. The IRT is bound up with thermal conductivity. With the influence of heat source, objects' surface will have some thermal spread. The difference of the thermal distribution can be used to detect the material difference under the objects' surface. IRT can image and orient horizontal plane and holes under the road efficiently.

Compare with other NDT methods, IRT has following advantages:

1. Fast inspection rate, which can up to a few $\mathrm{m}^{2}$ at a time;

2. No contact. No couplant needed although in some cases a black painting step is required to perform the inspection so that strictly speaking, a contact is thus present in this case;

3. Security of personnel. Since there is no harmful radiation involved, however high power external stimulation-such as powerful flashes-requires a shroud;

4. Results are relatively easy to interpret;

5. Unique inspection tool for some inspection tasks.

But on the other hand, there are some difficulties specific to IR thermography as follows:

1. Difficulty in obtaining a quick, uniform and highly energetic thermal stimulation over a large surface;

2. Effects of thermal losses (convective, radiative) which induce spurious contrasts affecting the reliability of the interpretation;

3. Cost of the equipment;

4. Capability of detecting only defects resulting in a measurable change of the thermal properties;

5. Ability to inspect a limited thickness of material under the surface;

6. Emissivity problems. 


\subsection{Radiography}

Radiography is a method that makes use of strong radiation, such as X-ray, to detect the inner situation of the object. Now this technique has been applied in the nondestructive test of the concrete. According to the sensitive photograph obtained from the steel bar and its defect inside the concrete, quantitative assessment is available by using the digital image analytical technique.

Besides, other imaging technique, such as ultrasonic imaging, interference imaging, is widely used in the test of road material. The application of these technique remarkably increases the quality of the road nondestructive testing, thus has an important role in enhancement of the development of our highway and transportation.

\section{The System and Mechanical Structure of the Mobile Robot}

\subsection{The System in Introduction}

The system structure of autonomous path planning mobile robot for road NDT shows in the Figure 1. The whole system using distributed control mode. First of all, used the GPS coordinate acquisition equipments to get the path information, then according to the coordinate information and the initial and final statement of the mobile robot, get robot motion curve function, and use the PC to figure out the kinematic parameter from the motion curve and kinematic model, send the parameter to the lower computer, which use AT89C51 single chip to control the step motors. Meanwhile, the NDT equipments on the robot sent the road information to the lower computer. After finish the whole route, the robot sent the road testing data back to the PC.

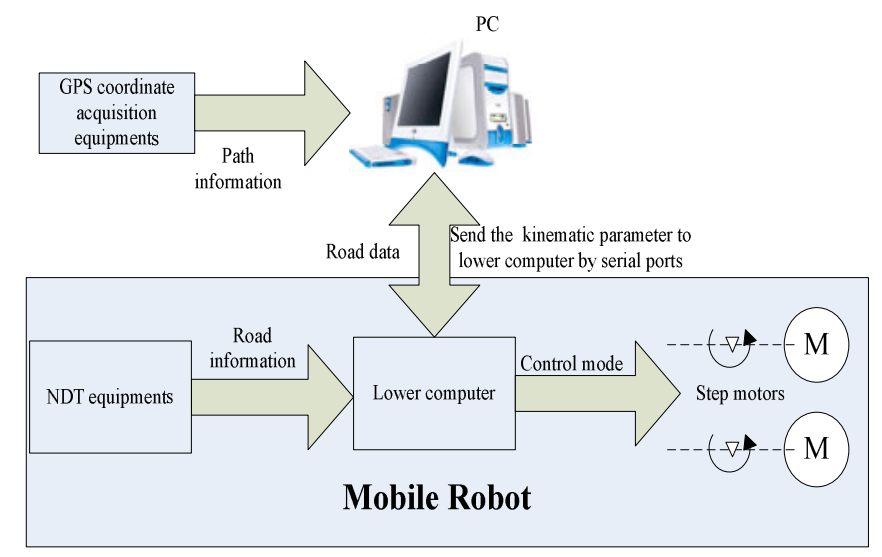

Fig. 1. The system structure of autonomous path planning mobile robot for road NDT

\subsection{The Structure of the Mobile Robot}

The research object of this paper is based on the mobile robot which is researched and designed by SCUT. The mobile robot use differential drive method, there are three 
wheels on the robot, two wheels are controlled by two step motors at front of the robot, which are as the drive wheels, the other rear wheel using flexible wheel at back to support the body of the robot. The moving direction is controlled by speed differential of the front two wheels.

\section{Fitting for the Path Moving Function}

Here, we use the regression equation to fit the moving function of the planed path. Regression equation is a statistical technique which can be used to predict the value of one variable using the value of one or a quantitative variable. According to the relationship between the two variables, regression equation can be divided into linear and nonlinear. According to the number of the variable, regression equation can be divided into unary and multiple.

\subsection{Linear Multiple Regression Equation}

Supposing $y$ is a random scalar, which has linear relationship with independent variables $x_{1}, x_{2} \ldots, x_{n}$, and according to the value of $n$ samples $\left(x_{i 1}, x_{i 2}, \ldots x_{i n} ; y_{i}\right)$ $(i=1,2, \ldots n)$, we can get the least squares estimation $\hat{b}_{0}, \hat{b}_{1}, \hat{b}_{2}, \ldots \hat{b}_{m}$. A multiple regression equation for predicting $\hat{y}$ can be expressed as follows:

$$
\hat{y}=\hat{b}_{0}+\hat{b}_{1} x_{1}+\hat{b}_{2} x_{2}+\ldots+\hat{b}_{m} x_{m}
$$

With $Q=\sum_{j=1}^{n}\left(y_{j}-\hat{y}_{j}\right)^{2}$

$$
=\sum_{j=1}^{n}\left(y_{j}-b_{0}-b_{1} x_{1 j}-b_{2} x_{2 j}-\cdots-b_{m} x_{m j}\right)^{2}
$$

Where $Q$ is the function of $b_{0}, b_{1}, b_{2}, \ldots, b_{m}$. According to the extreme value theory of multivariable function [7], when $Q$ get the minimum value:

$$
\left\{\begin{array}{l}
\frac{\partial Q}{\partial b_{0}}=-2 \sum_{j=1}^{n}\left(y_{j}-b_{0}-b_{1} x_{1 j}-b_{2} x_{2 j}-\cdots-b_{m} x_{n j}\right)=0 \\
\frac{\partial Q}{\partial b_{i}}=-2 \sum_{j=1}^{n} x_{i j}\left(y_{j}-b_{0}-b_{1} x_{1 j}-b_{2} x_{2 j}-\cdots-b_{m} x_{n j}\right)=0
\end{array} \quad(i=1,2 、 \ldots, m)\right.
$$

With above, (1) can be rewritten as:

$$
\left\{\begin{array}{l}
n b_{0}+b_{1} \Sigma x_{i 1}+b_{2} \Sigma x_{i 2}+\cdots+b_{m} \Sigma x_{i m}=\Sigma y_{i} \\
b_{0} \Sigma x_{i 1}+b_{1} \Sigma x_{i 1}^{2}+\cdots+b_{m} \Sigma x_{i 1} x_{i m}=\Sigma x_{1 i} y_{i} \\
\Sigma x_{2} b_{0}+b_{1} \Sigma x_{i 2} x_{i 1}+\cdots+b_{m} \Sigma x_{i 2} x_{i m}=\Sigma x_{i 2} y_{i} \\
\cdots \cdots \cdots \cdots \cdots \cdots \cdots \cdots \cdots \cdots \cdots \cdots \cdots \cdots \cdots \\
b_{0} \Sigma x_{i m}+b_{1} \Sigma x_{i m} x_{1}+\cdots+b_{m} \Sigma x_{i m}^{2}=\Sigma x_{i m} y_{i}
\end{array}\right.
$$


From the first equation of (4), we can get the following expression:

$$
\hat{b}_{0}=\bar{y}-\hat{b}_{1} \bar{x}_{1}-\hat{b}_{2} \bar{x}_{2}-\cdots-\hat{b}_{m} \bar{x}_{m}
$$

Substituting (5) into (4), we have

$$
\left[\begin{array}{c}
\hat{b}_{1} \\
\hat{b}_{2} \\
\vdots \\
\hat{b}_{m}
\end{array}\right]=\left[\begin{array}{llll}
l_{11} & l_{12} & \cdots & l_{1 m} \\
l_{21} & l_{22} & \cdots & l_{2 m} \\
l_{m 1} & l_{m 2} & \cdots & l_{m m}
\end{array}\right]^{-1}\left[\begin{array}{c}
l_{1 y} \\
l_{2 y} \\
\vdots \\
l_{m y}
\end{array}\right]
$$

Where:

$$
\left\{\begin{array}{l}
l_{u v}=\sum_{m}^{1}\left(x_{i u}-\bar{x}_{u}\right)\left(x_{i v}-\bar{x}_{v}\right) \\
l_{u y}=\sum_{m}^{1}\left(x_{i u}-\bar{x}_{u}\right)\left(y_{i}-\bar{y}\right)
\end{array} i, u, v=[1, m]\right.
$$

After this, the value of $\hat{b}_{0}$ can be got when substituting $\hat{b}_{1}, \hat{b}_{2}, \ldots \hat{b}_{m}$ into equation (5). So the linear multiple regression equation can be got as (1) shows.

\subsection{Nonlinear Regression Equation}

In statistics, nonlinear regression is a form of regression analysis in which observational data are modeled by a function which is a nonlinear combination of the model parameters and depends on one or more independent variables. The data are fitted by a method of successive approximations.

Some nonlinear regression problems, such as hyperbola regression model, logarithmic regression model and polynomial regression model can be moved to a linear domain by a suitable transformation of the model formulation [8]. But others have to deal with nonlinear regression model such as exponential model and $\mathrm{S}$ curve model.

\section{Kinematic Modeling for the Road NDT Mobile Robot}

Mobile robot kinematic modeling is the foundation of its motor system control. Accurate kinematic model plays a key role in the motion control system.

\subsection{Curve Fitting of Moving Mobile Robot}

It can be seen from Figure.2, when robot reached the point $\mathrm{n}$, the track for robot moving from point $\mathrm{n}$ to point $\mathrm{n}+1$ can be derived by fitting the robot moving function based on point $n$ to point $n+k$. In this paper, we takes $\mathrm{k}=5$, and only use 3 order polynomial regression models to fit the curve. So, the form of the moving function is as follow:

$$
y(n)=b_{0 n}+b_{1 n} x+b_{2 n} x^{2}+b_{3 n} x^{3}
$$


At this time, we can calculate the mobile control mode according to (7). When the robot reached point $n+1$ from point $n$, the new track for robot moving from point $\mathrm{n}+1$ to point $\mathrm{n}+2$ can be derived by fitting the robot moving function based on point $n+1$ to $n+k+1$. The form of the moving function is as follow:

$$
y(n+1)=b_{0(n+1)}+b_{1(n+1)} x+b_{2(n+1)} x^{2}+b_{3(n+1)} x^{3}
$$

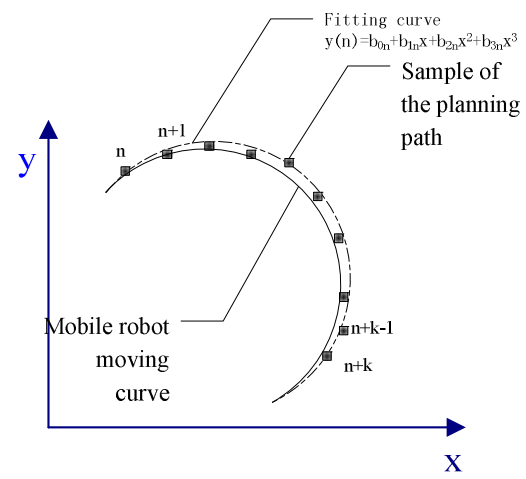

Fig. 2. Curve fitting of the mobile robot

Just like formula (7), we can calculate the mobile control mode according to formula (8), and so on.

As above done, the mobile robot can move along the track which is planned beforehand by adjusting the robot curve.

\subsection{Moving Modeling for the Mobile Robot}

Figure. 3 shows that the mobile robot's moving curve is a circle with radius $\mathrm{R}$.

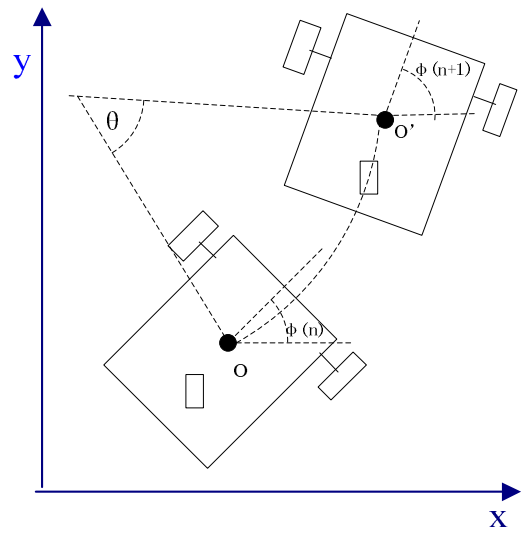

Fig. 3. Mobile robot's moving curve is a circle with radius $R$ 
Suppose that at the moment $\mathrm{n}$, the robot's centroid is at the point $\mathrm{O}$, the coordinate of $\mathrm{O}$ is $\left(x_{n}, y_{n}\right)$, and the angel of the robot and the horizontal plane is $\varphi(n)$. In the ideal case, the direction of the moving velocity is the same as the moving curve tangent line. So:

$$
d x_{n} \sin \varphi_{n}-d y(n) \cos \varphi_{n}=0
$$

from (9), we can get:

$$
\tan \varphi_{n}=\frac{\sin \varphi_{n}}{\cos \varphi_{n}}=\frac{d y(n)}{d x_{n}}
$$

At the moment of $n+1$, the mobile robot moves from $\mathrm{O}$ to $\mathrm{O}^{\prime}$, and the coordinate of $\mathrm{O}^{\prime}$ is $\left(x_{n+1}, y_{n+1}\right)$, and the angel between the robot and the horizontal plane is $\varphi(n+1)$.

As shown in Figure.3, $\theta$ is the turning angel of the mobile robot, according to the lane Analytic Geometry [9], we can get:

$$
\theta=\varphi_{n+1}-\varphi_{n}=\arctan \frac{d y(n+1)}{d x_{n+1}}-\arctan \frac{d y(n)}{d x_{n}}
$$

Use the Pythagorean theorem, we can get:

$$
\Delta x^{2}+\Delta y^{2}=\left(2 R \sin \frac{\theta}{2}\right)^{2}
$$

Then

$$
R= \begin{cases}\frac{\sqrt{\Delta x^{2}+\Delta y^{2}}}{2 \sin \frac{\theta}{2}} & \theta \neq 0 \\ +\infty & \theta=0\end{cases}
$$

Where

$$
\left\{\begin{array}{l}
\Delta x=x_{n+1}-x_{n}=2 R \sin \frac{\theta}{2} \cos \left(\frac{\theta}{2}+\varphi_{n}\right) \\
\Delta y=y_{n+1}-y_{n}=2 R \sin \frac{\theta}{2} \sin \left(\frac{\theta}{2}+\varphi_{n}\right)
\end{array}\right.
$$

\section{Method of Calculating the Kinematic Parameter for the Mobile Robot}

Kinematic system is one of the important core problems and technique in the research area of differential drive mobile robot control system, and the moving curve of the robot is decided by the robot moving system.

Generally speaking, all the moving tracks of the robot are made up of straight lines and curves. So even if the robot moves in different route, the control method is the 
same. Just like above, the robot's moving tracks can be control accuracy by controlling the ratio of the two driving wheels well.

\subsection{The Kinematic Parameter of the Robot When Straight-Line Moving}

As shown in Figure.4, in the case of straight-line moving, $\varphi_{n+1}=\varphi_{n}, \theta=0$, and the speed of the left wheel is the same as the right one. If taking the speed ratio of the left wheel and right wheel as $k$, then $k=1$ in this case.

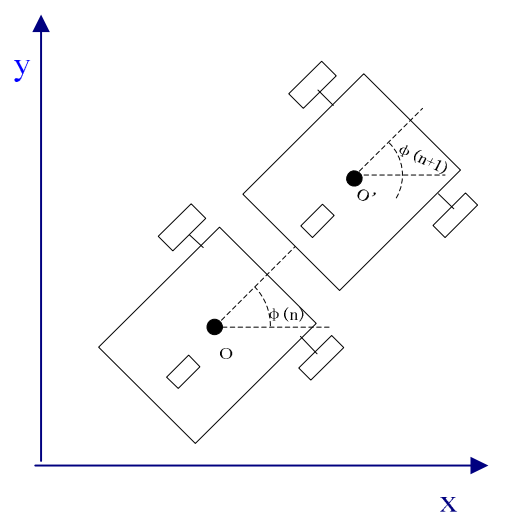

Fig. 4. The robot is in the case of straight-line moving

At this time, the distance of the robot moved is:

$$
S=\frac{S_{1}+S_{2}}{2}=S_{1}=S_{2}
$$

In (15), $S_{1}$ is the distance the left wheel moved, and $S_{2}$ is the right one.

\subsection{The Kinematic Parameter of the Robot When Curvilinear Moving}

As shown in the Figure.3, in the case of curvilinear moving, when the robot is turning left, $\varphi_{n+1} \neq \varphi_{n}, \theta \neq 0$ the speed of the left wheel is not the same as the right one. So, at this time, from the meaning of $k$, we can get:

$$
k=\frac{v_{1}}{\mathrm{v}_{2}}=\frac{v_{1} \cdot \mathrm{t}}{\mathrm{v}_{2} \cdot \mathrm{t}}=\frac{S_{1}}{\mathrm{~S}_{2}}=\frac{(R-b) \theta}{R \theta}=1-\frac{b}{R}
$$

Where: $v_{1}$ the speed of the left wheel;

$S_{1}$ — the distance of the left wheel moved

$v_{2}$ the speed of the right wheel;

$\mathrm{S}_{2}$ the distance of the right wheel moved;

$b$ the distance from the left wheel to the right one. 
Use (13) input (16) can get:

$$
k=1-\frac{2 b \sin \frac{\theta}{2}}{\sqrt{\Delta x^{2}+\Delta y^{2}}}
$$

Use (11) input (17) can get:

$$
k=1-\frac{2 b \sin \left[\arctan \frac{d y(n+1)}{d x_{n+1}} / 2-\arctan \frac{d y(n)}{d x_{n}} / 2\right]}{\sqrt{\Delta x^{2}+\Delta y^{2}}}
$$

When the robot is turning right:

$$
k=\frac{v_{1}}{\mathrm{v}_{2}}=\frac{v_{1} \cdot \mathrm{t}}{\mathrm{v}_{2} \cdot \mathrm{t}}=\frac{S_{1}}{\mathrm{~S}_{2}}=\frac{(R+b) \theta}{R \theta}=1+\frac{b}{R}=1+\frac{2 b \sin \frac{\theta}{2}}{\sqrt{\Delta x^{2}+\Delta y^{2}}}
$$

Use (11) input (19) can get:

$$
k=1+\frac{2 b \sin \left[\arctan \frac{d y(n+1)}{d x_{n+1}} / 2-\arctan \frac{d y(n)}{d x_{n}} / 2\right]}{\sqrt{\Delta x^{2}+\Delta y^{2}}}
$$

When the mobile robot is curvilinear moving, the distance of the robot moved is:

$$
S=\frac{S_{1}+S_{2}}{2}=\frac{(R-b) \theta+(R+b) \theta}{2}=R \theta
$$

\section{Example}

In order to prove the accuracy of the model set above, we used the mobile robot and PC to simulate this kinematic model. Fist of all, got the information of the moving path, the sampling of the coordinate information is as shown in Figure.5.

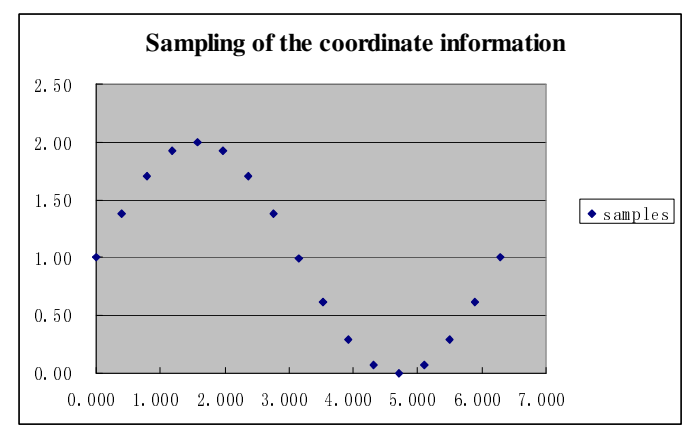

Fig. 5. Sampling of the coordinate information 
Then input the initial statement of the mobile robot which is $\left(x_{0}, y_{0}, \varphi_{0}\right)=(0,0,0)$, and the final statement is $\left(x_{n}, y_{n}, \varphi_{n}\right)=\left(2 \pi, 1, \frac{\pi}{2}\right)$.we got the distance from the left wheel to the right one is $b=0.295 \mathrm{~m}$, the radius of the drive wheels is $r=0.0375 m$.

First of all, use nonlinear multiple regression equation to fit out the next moving function according to the next 5 points' coordinates, The least squares estimation parameters $\hat{b}_{0}, \hat{b}_{1}, \hat{b}_{2}, \hat{b}_{3}$, as shown in Tab 1 :

Table 1. Fitting out the moving function

\begin{tabular}{|c|c|c|c|c|}
\hline Function & $\mathrm{b} 0$ & $\mathrm{~b} 1$ & $\mathrm{~b} 2$ & $\mathrm{~b} 3$ \\
\hline 1 & 1 & 1.02 & -0.07 & -0.11 \\
2 & 0.97 & 1.15 & -0.22 & -0.06 \\
3 & 0.83 & 1.48 & -0.47 & 0 \\
4 & 0.53 & 2.04 & -0.8 & 0.06 \\
5 & 0.03 & 2.75 & -1.13 & 0.11 \\
6 & -0.52 & 3.43 & -1.4 & 0.15 \\
7 & -0.83 & 3.75 & -1.51 & 0.16 \\
8 & -0.4 & 3.35 & -1.39 & 0.15 \\
9 & 1.34 & 1.92 & -1 & 0.11 \\
10 & 4.91 & -0.71 & -0.36 & 0.06 \\
11 & 10.47 & -4.44 & 0.47 & 0 \\
12 & 17.63 & -8.85 & 1.37 & -0.06 \\
13 & 25.32 & -13.2 & 2.2 & -0.11 \\
\hline
\end{tabular}

After this, use the kinematic model to get the kinematic parameters; the PC simulates picture shows in the Figure.6.

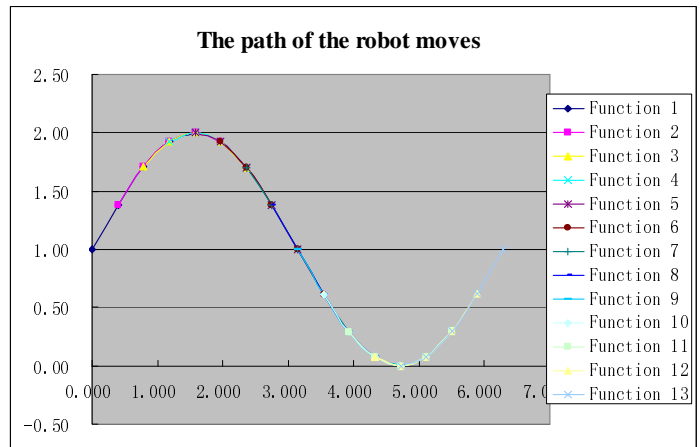

Fig. 6. The path of the robot moves 
Because of the road friction, there is error exist during the testing. But the error is less than $0.2 \%$. So, the model can be thought accuracy.

\section{Conclusion}

This paper analyses the situation about the methods of video imaging, CT, infrared thermography and radiography applied in the road nondestructive testing. Besides, appropriate algorithm for nondestructive testing on the road autonomous mobile robot path planning is given .The feasibility of the scheme and algorithm proposed in this paper was verified by the experimental prototype test data.

\section{References}

1. Shi, P.F., Zhao, Y.G.: A Survey of Environment Modeling in Mobile Robot: Design and Research, China, pp.1-6 (January 2010) (in Chinese)

2. Zhang, J., Sha, A.M., Sun, Z.Y.: The Application of Digital Image Processing to Road Nondestructive Test. Shanxi Science and Technology of Communications, Shanxi, pp. 1012 (December 2002) (in Chinese)

3. Cheng, H.D., Miyojin, M.: Automatic pavement distress detection system: Information Sciences, pp. 219-240. Elsevier Science, Amsterdam (1998) (in Chinese)

4. Chang, Y., Ma, S.G., Wang, H.G.: Method of Kinematic Modeling of Wheeled Mobile Robot. Journal of Mechanical Engineering, 30-35 (March 2010) (in Chinese)

5. Kim, D.H., Jun-Hooh: Globally Asymptotically Stable Tracking Control of Mobile Robots. In: The 1998 IEEE International Conference on Control Applications, pp. 305-307 (1998)

6. Addison, D.: Introduction to Robotics Technology. Developer works, USA (2001)

7. Fiorini, P., Shiller, Z.: Time Optimal Trajectory Planning in Kinematic Environments. Journal of Applied Mathematics and Computer Science, USA (1997)

8. Zuang, C.Q., He, C.X.: Statistics Statistical. South China University of Technology Press, Guangzhou (2006) (in Chinese)

9. Zuang, C.Q., Yao, Y.X., Luo, J.H.: Higher Mathematics. South China University of Technology Press, Guangzhou (2007) (in Chinese) 